Please do not remove this page

RMIT

UNIVERSITY

\title{
A Landscape-Scale Optimisation Model to Break the Hazardous Fuel Continuum While Maintaining Habitat Quality
}

Leon Caballero, Javier; Reijnders, Victor; Hearne, John; Ozlen, Melih; Reinke, Karin

https://researchrepository.rmit.edu.au/esploro/outputs/9921863392001341/filesAndLinks?institution=61RMIT_INST\&index=null

Leon Caballero, J., Reijnders, V., Hearne, J., Ozlen, M., \& Reinke, K. (2019). A Landscape-Scale Optimisation Model to Break the Hazardous Fuel Continuum While Maintaining Habitat Quality. Environmental Modeling and Assessment, 24(4), 369-379. https://doi.org/10.1007/s10666-018-9642-2

Document Version: Accepted Manuscript

Published Version: https://doi.org/10.1007/s10666-018-9642-2

Repository homepage: https://researchrepository.rmit.edu.au

(C) Springer Nature Switzerland AG 2018

Downloaded On 2023/04/27 01:30:24 +1000 
Thank you for downloading this document from the RMIT Research Repository.

The RMIT Research Repository is an open access database showcasing the research outputs of RMIT University researchers.

RMIT Research Repository: http://researchbank.rmit.edu.au/

\section{Citation:}

Leon Caballero, J, Reijnders, V, Hearne, J, Ozlen, M and Reinke, K 2019, 'A landscape-scale optimisation model to break the hazardous fuel continuum while maintaining habitat quality', Environmental Modeling and Assessment, vol. 24, no. 4, pp. 369-379.

See this record in the RMIT Research Repository at:

https://researchbank.rmit.edu.au/view/rmit:55229

Version: Accepted Manuscript

Copyright Statement:

(C) Springer Nature Switzerland AG 2018

\section{Link to Published Version:}

https://dx.doi.org/10.1007/s10666-018-9642-2 


\title{
A landscape-scale optimisation model to break the hazardous fuel continuum while maintaining habitat quality
}

\author{
Javier León ${ }^{1,3}$, Victor M.J.J. Reijnders ${ }^{1,2}$, John W. Hearne ${ }^{1, *}$, Melih Ozlen $^{1}$ and \\ Karin J. Reinke ${ }^{1}$

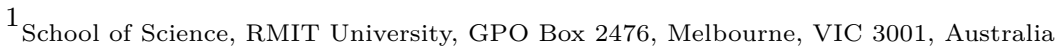 \\ ${ }^{2}$ Department of Applied Mathematics, University of Twente, P.O. Box 217, 7500 AE Enschede, The Netherlands \\ ${ }^{3}$ Faculty of Mathematical Sciences, Complutense University of Madrid, Plaza de las Ciencias 3, 28040 Madrid, Spain
}

September 28, 2018

\begin{abstract}
Wildfires have demonstrated their destructive powers in several parts of the world in recent years. In an effort to mitigate the hazard of large catastrophic wildfires a common practice is to reduce fuel loads in the landscape. This can be achieved through prescribed burning or mechanically. Prioritising areas to treat is a challenge for landscape managers. To help deal with this problem we present a spatially explicit, multi-period mixed integer programming model. The model is solved to yield a plan to generate a dynamic landscape mosaic that optimally fragments the hazardous fuel continuum while meeting ecosystem considerations. We demonstrate that such a multi-period plan for fuel management is superior to a myopic strategy. We also show that a range of habitat quality values can be achieved without compromising the optimal fuel reduction objective. This suggests that fuel management plans should also strive to optimise habitat quality. We illustrate how our model can be used to achieve this even in the special case where a faunal species requires mature habitat that is also hazardous from a wildfire perspective. The challenging computational effort required to solve the model can be overcome with either a rolling horizon approach or lexicographically. Typical Australian heathland landscapes are used to illustrate the model but the approach can be implemented to prioritize treatments in any fire-prone landscape where preserving habitat connectivity is a critical constraint.
\end{abstract}

\section{Keywords}

Wildfires, spatial optimisation, fuel continuum, habitat quality, multiperiod landscape planning, mixed integer programming

\section{Introduction}

Although some negative effects have been noted, positive effects of bush fires on the habitat for native flora and fauna have been recorded [40]. Reports indicate that areas subject to prescribed burning have more live trees, greater survival, and reduced fire intensity during wildfires compared to untreated areas [38]. Thinning and burning has also been employed for restoration purposes [2]. Prescribed burning leads to fuel reduction [1] and areas with old vegetation (or areas with excess fuel build-up) are often targeted for treatment [17]. Treatments may restrict the spread and intensity of large wildfires and so help mitigate wildfire hazards [36, 5, 8], and the risk to human life and economic assets [29]. Thus it has been argued that fuel management is both necessary and important [7]. 
The spatial configuration of fuel in the landscape has been explored to assess its effect on fire spread [21, 35]. Patterns include disconnected fuel treatment patches that overlap in the direction of fire spread [14], and taking into account the surrounding natural landscape [15]. An analysis involving extensive simulations looked at the effectiveness of fuel-break networks [28]. Also preparing explicitly for possible future fires when choosing where to apply treatment [41] taking into account fire occurrence, major fire-flow paths and burn probabilities [43]. Stochastic programming with sample fires has produced some spatial and temporal relationships for where to burn [27].

The occurrence of catastrophic wildfires decreases with extent treated [23, 22] but with an optimal landscape mosaic [16] hazard reduction can be achieved without excessive costs [24]. Nevertheless the vegetation regenerates, senesces and eventually becomes high fuel load again and treatment extent is always subject to a budget constraint. In Mediterranean climate systems the wet winters and the dry and dangerous conditions in summer further restrict prescribed burning to a narrow time-window. These constraints on treatment extent can lead to desired management plans becoming infeasible in some years. Thus long-term planning is necessary to minimise high-fuel load connections over time [42, 26, 31].

Reducing the total fuel load has ecological consequences. Some species rely on vegetation that is classified as high-fuel load. A native Australian bird, the Mallee Emu-wren, is an example [6] and the Southern Brown Bandicoot requires heathland that was last burnt between five and fifteen years ago [37]. Treatment plans should take into account the habitat needs of species such as these. This might require sufficient areas of high fuel-load to remain after treatment. Prioritising areas for prescribed burning would then involve trying to disrupt fire-spread paths while, for example, maintaining migration paths of fauna [33]. Little research has been done combining multiple concerns that arise with fuel treatment in an optimisation framework [10].

For management purposes states are often divided into large planning areas such as a catchment or national park. Each of these planning areas is then divided into treatment units or potential operational delineations (PODs)[39]. These areas are often determined by boundaries such as roads or rivers which facilitates the control of prescribed fire or some other management activities. Formulating a multiperiod schedule of treatments for a landscape comprising such treatment units is a complex spatio-temporal problem [19, 34] and the resulting landscape mosaic is critical for hazard reduction $[13,23]$ and habitats. An early model addressing the fuel hazard problem only was formulated and illustrated on a regular grid [26]. The approach was then extended to include multiple vegetation classes in a real landscape [32]. The computational effort in this work limited the analysis to some extent. More recently a study involving much greater emphasis on the probability of fire occurrence, fire behaviour and assets at risk was published [3]. This work is aimed at prioritising treatment in preparation for the next fire season and does not deal with the multiperiod problem. In another recent work [30] a multiperiod model for fuel management was constructed. that comprised ecological constraints. These included the vegetations' "tolerable fire intervals" [9] as well as the quantity and spatial configuration of habitat for a faunal species. In this paper we advance the previous model [30] in the following way:

- the rectangular grid structure is replaced by more realistic polygons

- the concept of connectedness is broadened to recognise the length of a common boundary

- the concept of neighbourhood is changed to reflect the direction of fire spread or migration paths

- fuel accumulation curves are used to categorise hazardous polygons [20] 
- fire response curves are used to generate a measure of habitat quality of a polygon [25]

- limitations imposed by computational effort are overcome to some extent by either a rolling horizon or a lexicographic approach to the solution method

The model we formulate is a mixed integer programming model with the aim of finding an optimal multiperiod, spatial schedule for prescribed burning that will:

- reduce the connectivity of high fuel load polygons in order to lessen the likelihood of large wildfires

- ensure the sustainability of the vegetation by constraining the timing of prescribed burns within tolerable fire intervals

- ensure adequate levels of habitat quality for fauna are maintained

\section{Materials and Method}

\section{Model Description and formulation}

Consider a landscape (i.e. a management area) comprising a mosaic of polygons. In the context of fuel management these polygons are often referred to as 'treatment units' or 'burn units'. The time since the vegetation in each polygon was last burnt determines its fuel load. For convenience for the rest of this paper we will refer to the time elapsed since the vegetation in each polygon was last burnt as its "age". Vegetation age also characterises the habitat suitability for particular fauna of each polygon $[6,37]$. In this model we consider a single vegetation type (heathland) and without specifying a species we consider invertebrates that prefer some predefined vegetation age [11]. We formulate a model that each year selects the polygons to undergo fuel reduction through controlled burning or mechanical clearing. The sequence of selections is made so as to minimise a metric for wildfire hazard. This is achieved by ensuring that after treatment the polygons remaining with high fuel load are as fragmented as possible.

On the other hand we also want to take into account the species that live in the landscape. As species have preferences for vegetation of a certain age [11], we assign a quality to each polygon according to its area and the relative abundance of species supported by vegetation of that age. We can then only select a polygon for treatment if the habitat quality of at least one of its neighbours is at least as good as the habitat quality of the polygon itself. This way, we take into account the habitat needs of the species, although we realize that individuals might have to migrate from time to time.

Further constraints included in the model relate to the vegetation. To sustain vegetation and the associated ecosystem, fire should not occur more frequently than its 'minimum tolerable fire interval'. On the other hand, for fire-dependent species the 'maximum tolerable fire interval' is also important [9]. An explanation of all constraints is described after the mathematical representation of the model that follows. 
Sets:

$I \quad$ is the set of all burn units in the landscape

$\Phi_{i} \subset I \quad$ is the set of burn units connected to burn unit $i$

Parameters:

$a_{i} \quad$ initial fuel age of burn unit $i$

$b_{t} \quad$ fuel treatment budget at time $t$

$c_{i} \quad$ area of burn unit $i$

$w_{i j} \quad$ relative weighting of the connectivity of burn units $i$ and $j$

$h_{t h r} \quad$ high-fuel load threshold

$h_{\text {targ }} \quad$ global habitat quality target

maxTFI maximum tolerable fire interval (TFI)

minTFI minimum tolerable fire interval (TFI)

$T \quad$ number of time periods in the planning horizon

$N \quad$ number of breakpoints for the piecewise linear function of the fire response curve

$r_{n} \quad$ breakpoint of the piecewise linear function or the fire response curve

$v_{n} \quad$ value of breakpoint $r_{n}$ according to the fire response curve

$p_{i j} \quad$ proportion of boundary of unit $i$ that is shared with unit $j$

$M \quad$ big-M coefficient

Variables:

$X_{i t} \quad 1$ if burn unit $i$ is treated in time period $t, 0$ otherwise

$H_{i t} \quad 1$ if burn unit $i$ is classified as high-fuel load in time period $t, 0$ otherwise

$Q_{i j t} \quad 1$ if adjacent burn units $i$ and $j$ are both classified as high-fuel load in time period $t, 0$ otherwise

$A_{i t} \quad$ fuel age of burn unit $i$ in time period $t$

$F R C_{i t} \quad$ habitat quality of burn unit $i$ in time period $t$ by area (fire response curve)

$Z_{i t n} \quad 1$ if the age of burn unit $i$ in time period $t$ is between $r_{n}$ and $r_{n+1}, 0$ otherwise

$G_{i t n} \quad$ convex multipliers of burn unit $i$ in time period $t$ for the piecewise linear function 
Minimise $\sum_{t=2}^{T} \sum_{i \in I} \sum_{\substack{j \in \Phi_{i} \\ j>i}} w_{i j} Q_{i j t}$

Subject to:

$$
\begin{aligned}
& \sum_{i \in I} c_{i} X_{i t} \leq b_{t} \\
& t=2, \ldots, T \\
& t=1 \quad \forall i \in I \\
& A_{i t}=a_{i} \\
& A_{i t} \geq A_{i(t-1)}+1-M \cdot X_{i t} \\
& A_{i t} \leq A_{i(t-1)}+1 \\
& A_{i t} \leq \operatorname{maxTFI}\left(1-X_{i t}\right) \\
& \operatorname{minTFI} \cdot X_{i t} \leq A_{i(t-1)} \\
& A_{i t} \leq h_{t h r}-1+M \cdot H_{i t} \\
& H_{i t}+H_{j t} \leq 1+Q_{i j t} \\
& \sum_{n=1}^{N-1} Z_{i t n}=1 \\
& Z_{i t n} \leq G_{i t n}+G_{i t(n+1)} \\
& \sum_{n=1}^{N} r_{n} G_{i t n}=A_{i t} \\
& t=2, \ldots, T \quad \forall i \in I \\
& t=2, \ldots, T \quad \forall i \in I \\
& t=2, \ldots, T \quad \forall i \in I \\
& t=2, \ldots, T \quad \forall i \in I \\
& t=2, \ldots, T \quad \forall i \in I \\
& t=2, \ldots, T \quad \forall i \in I \quad \forall j \in \Phi_{i}, j>i \\
& t=1, \ldots, T \quad \forall i \in I \\
& t=1, \ldots, T \quad \forall i \in I \quad n=1, \ldots, N-1 \\
& t=1, \ldots, T \quad \forall i \in I \\
& \sum_{n=1}^{N} G_{i t n}=1 \\
& t=1, \ldots, T \quad \forall i \in I \\
& F R C_{i t}=\sum_{n=1}^{N} v_{n} G_{i t n} \\
& c_{i} F R C_{i(t-1)}-M\left(1-X_{i t}\right) \leq \sum_{j \in \Phi_{i}} p_{i j} c_{j} F R C_{j t} \\
& t=1, \ldots, T \quad \forall i \in I \\
& t=2, \ldots, T \quad \forall i \in I \\
& \sum_{i \in I} c_{i} F R C_{i t} \geq h_{t a r g} \\
& t=2, \ldots, T \\
& X_{i t} \in\{0,1\} \\
& t=2, \ldots, T \quad \forall i \in I \\
& H_{i t} \in\{0,1\} \\
& t=2, \ldots, T \quad \forall i \in I \\
& t=1, \ldots, T \quad \forall i \in I \quad n=1, \ldots, N-1 \\
& t=2, \ldots, T \quad \forall i \in I \quad \forall j \in \Phi_{i}, j>i \\
& t=1, \ldots, T \quad \forall i \in I \\
& t=1, \ldots, T \quad \forall i \in I \\
& t=1, \ldots, T \quad \forall i \in I \quad n=1, \ldots, N
\end{aligned}
$$

The objective function (1) minimises the weighted number of connections between high-fuel load burn units. This dimensionless quantity will henceforth be referred to as fire hazard. Constraint (2) limits the amount of land we can burn each year. Constraint (3) initializes the ages of all burn units and constraints (4) - (6) track the age of all burn units, resetting the age to 0 if we burn the unit. Constraint (6) forces a unit to be burnt if its age is equal to the maximum TFI 
and constraint (7) only allows a unit to be burnt if its age is over the minimum TFI. Constraint (8) ensures a burn unit to be classified as high-fuel if the age of burn unit is equal to or larger than a threshold value $h_{t h r}$. Constraint (9) sets $Q_{i j t}$ to 1 if both burn units $i$ and $j$ are classified as high-fuel load in time period $t$.

Constraints (10) - (13) model the piecewise linear function of the fire response curve, which is dependent on the age $A_{i t}$. We break the original curve at breakpoints $r_{n}$ which have value $v_{n}$. Constraint (10) is used so that the age can only be at one linear function at a time. Constraint (11) forces the model to only use the convex multipliers of the corresponding linear function. Constraint (12) and (13) makes sure the age is a convex combination of the breakpoints. Then constraint (14) ensures $F R C_{i t}$ is given the correct value according to the piecewise linear function.

Constraint (15) ensures unit $i$ can be burnt at time $t$ only if the habitat quality in the neighbourhood of unit $i$ is at least as good as the quality that unit $i$ had at time $t-1$. To enforce this constraint at $t=2$ values of $F R C_{i t}$ are required at $t=1$, hence the summations in equations (10-14) commence at $t=1$. The quality of the neighbourhood, henceforth referred to as local quality takes into account both the areas of neighbouring units as well as the proportion of common boundary. Constraint (16) ensures that global habitat quality is maintained above some target level. We use the term 'global habitat quality' to mean the area-weighted sum of habitat quality over the whole landscape.

Constraints (17) - (19) ensure the decision variables are binaries and constraints (20) - (23) restrict the age and quality of a unit to only positive values, as well as the breakpoint values.

By definition $Q_{i j t}=Q_{j i t}$, and since the weighting of a connection between two cells is determined by the relative length of their common boundary $w_{i j}=w_{j i}$, and hence $w_{i j} Q_{i j t}=$ $w_{j i} Q_{j i t}$. Thus the number of binary variables can be reduced by excluding all $Q_{i j t}$ such that $i \leq j$. This saves computational effort and will have no impact on the optimal strategy as it effectively involves dividing the objective function by a constant.

Also note that the initial conditions are set at $t=1$. The first decision on where to burn is based on these initial conditions but the consequences of that decision is only realised at $t=2$. Thus fire hazard is tracked and accounted for in the objective function from year 2 .

\section{Model Implementation}

\subsection{Implementation}

The model is implemented for a heathy woodland vegetation type with 23 randomly generated landscapes (one instance is shown in Figure 1). Each of the landscapes has 45 burn units. The average size of a burn unit in this implementation is taken to be 100 ha but within certain limits the representation is scale-free. Experiments are performed with a treatment level of 7 percent of the total area of the landscape each year. The simulations are solved for a planning period of 20 years, with a rolling horizon of 12 years. The problems are solved on a PC using Gurobi 7.5 [18] with the JuMP modeller [12] and the Julia programming language [4]. 


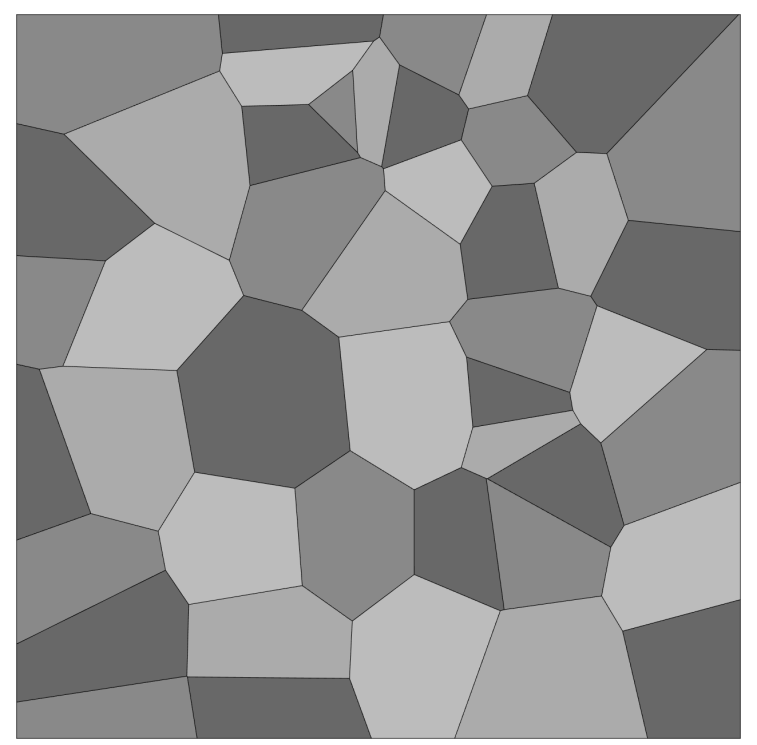

Figure 1: Randomly generated landscape with 45 polygons representing a planning area of 45 $\mathrm{km}^{2}$. On average polygons are 100 ha in extent.

\subsection{Input data}

Based on [9] the appropriate parameters for heathland are as follows: $h_{t h r}=10, \operatorname{minTFI}=10$, and $\operatorname{maxTFI}=35$. The maxTFI parameter suggested [9] is 45 rather than 35 years. However, as seen in Figure 2 any unit with a fuel age over 35 years has no habitat value and also represents high fuel hazard. Thus, to improve computation time we have set the parameter maxTFI to be equal to 35 .

Habitat considerations are illustrated by the needs of invertebrates in the vegetation. The habitat quality of burn unit $i$ at time $t$ is given by the product of its area and its age-related quality. Based on [11] the latter is given by the piecewise linear function shown in Figure 2.

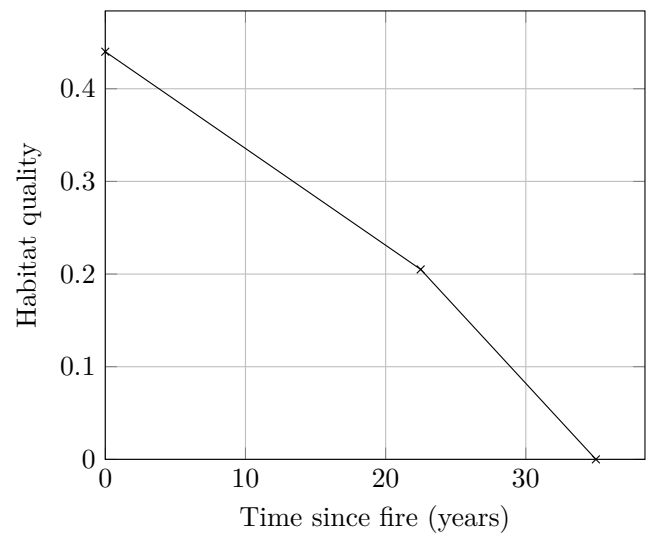

Figure 2: Habitat quality as a function of fuel age. Values fitted with data from [11] for the optimal age distribution for invertebrates on a heathy woodland landscape. 
The initial value of global habitat quality is chosen as the target value $h_{\text {targ }}$ in constraint (16) of the model. This ensures that there is no decline in global habitat quality throughout the simulation period.

\subsection{Experiments}

\section{Rolling horizon}

Solutions are obtained over 20 years using a rolling horizon of 12 years for the following cases:

rh1: The model presented above is solved to give the nomimal solution.

rh2: The local habitat constraints are relaxed and compared with the nominal solution to determine what effect they have on fire hazard.

rh3: The model is solve with a short-term planning period (two-year rolling horizon) and compared with the nominal solution to determine the importance of a longer-term planning. Habitat constraints are dropped to enable a clearer comparison of fire hazard with the two planning period approaches.

\section{Lexicographic approach}

The following solutions were obtained for a planning period of 20 years using a lexicographic approach. Using this approach solutions over the full 20-year planning period can be obtained without requiring a rolling horizon. Further changes made include the following:

- The habitat quality curve shown in Figure 2 is changed to create greater tension between reducing fuel load and maintaining habitat quality. The new habitat quality curve is shown in Figure 3.

- In previous experiments fire hazard was defined to be the sum of fire hazard over the full planning period. In this experiment we consider an alternative metric, which might be considered more appropriate by fire managers. We redefine the total fire hazard to be the highest fire hazard in any year. Hence we minimize over the planning period the maximum fire hazard that can occur in any given year (minimax approach). As fire hazard in the first few years is more a reflection of random initial values than any plan, we only consider the second half of the planning horizon.

- As with fire hazard, total habitat value was defined to be a sum throughout the planning horizon. We also redefine total habitat value to be the minimum habitat value in any year, starting in the middle of the planning horizon (maximin approach). This new approach is easily justified when we consider that an animal living on the landscape would hardly benefit from a solution in which some years have very high habitat value but other years have a habitat value close to zero. 


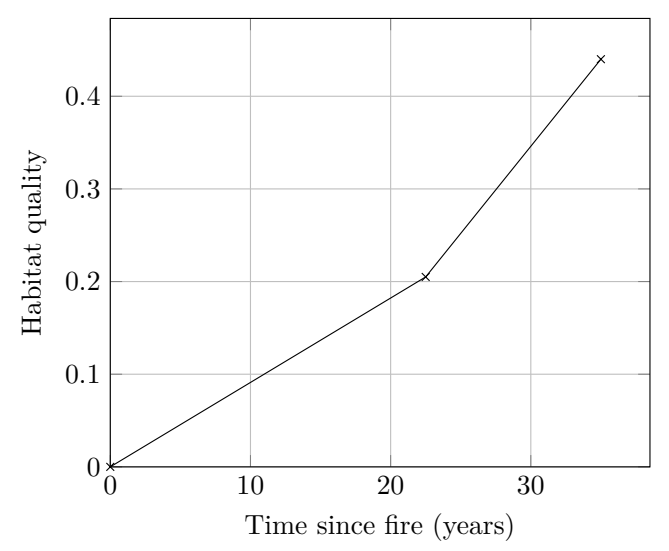

Figure 3: Fire response curve: habitat quality of a unit given its age (by hectare)

L1: To understand what range of global habitat values might result when minimising fuel hazard the following sequence was undertaken:

- Relax habitat constraints and then minimise the fuel hazard to yield an optimal fuel objective value $f_{m}$.

- Without compromising $f_{m}$, maximise the habitat value to yield an optimal habitat value $h_{M}$

- Still maintaining the fuel hazard at $f_{m}$, minimise the habitat value to yield $h_{m}$.

If fuel hazard reduction is a priority for landscape managers, the interval $\left\{h_{m}, h_{M}\right\}$ will show the extent of habitat outcomes possible without compromising fuel hazard objectives.

L2: In the case where a key faunal species requires habitat with a high fuel load it might be important to ascertain differences between fire paths and possible directions of local migration of fauna. To represent these paths the neighbourhood data must be amended accordingly. Our model can easily reflect different neighbourhood definitions. For example a landscape could be located in some place where wind primarily blows in one direction, and hence fire propagation would occur mainly in that direction. If that were the case our model can reflect that information by just changing a neighbourhood matrix. In the model formulation the neighbourhood information is given by the set $\left.\Phi_{i}\right)$. An example of this alternative way of defining neighbours is shown in Figure 4. Another example where fire propagation might occur mainly in one direction (and thus neighbourhoods defined in a similar way) is if the landscape has a high slope and fires are primarily topographical. 


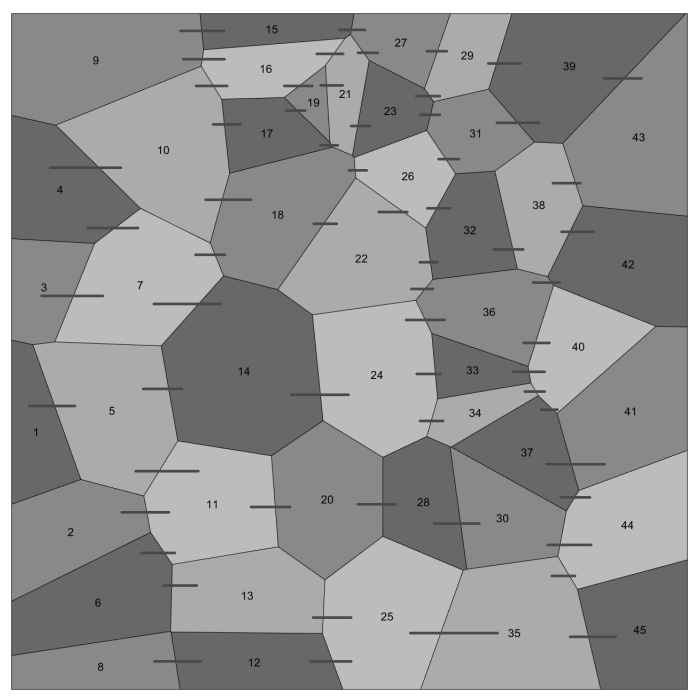

Figure 4: If the landscape has prevailing winds in the west-east direction, and fires are wind-driven, the neighbourhood matrix can reflect this. For this case lines in the figure show which units are defined as neighbours.

With the neighbours defined as given by Figure 4 we solve the model lexicographically.

\section{Results and Discussion}

For experiment(rh1) the mean fire hazard and global habitat value are shown in Figure 5.

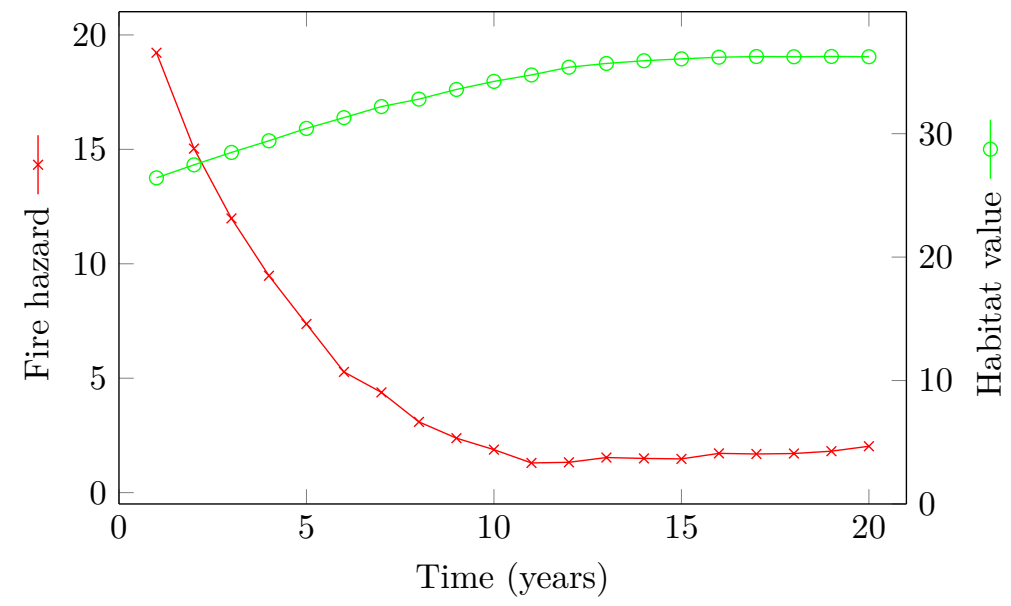

Figure 5: Mean fire hazard and mean global habitat value for the 23 scenarios by year

Our objective was to get an overall minimum in the weighted connections between high-hazard burn units. We see that the initial hazard is quickly brought close to 0 , while maintaining habitat of good quality (both local and global). (Figures 6, 7 and 8) show the initial conditions (random ages) and the solution after 3 and 19 years. It is clear that the model is achieving its aim. 


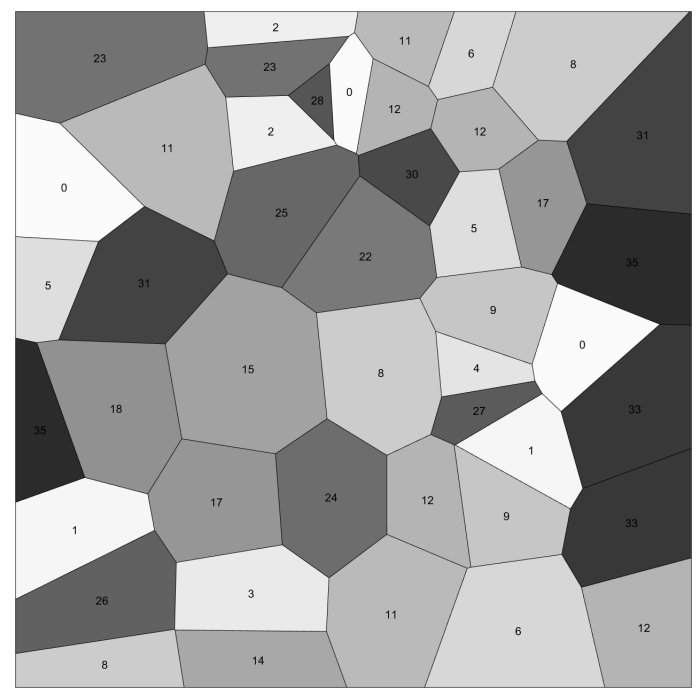

Figure 6: Ages of cells on random initial conditions for a given landscape

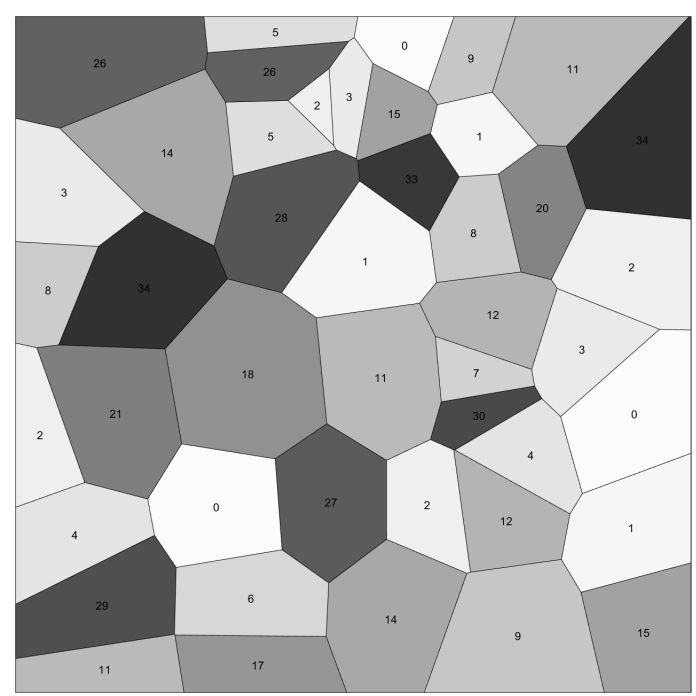

Figure 7: Ages of cells after 3 years 


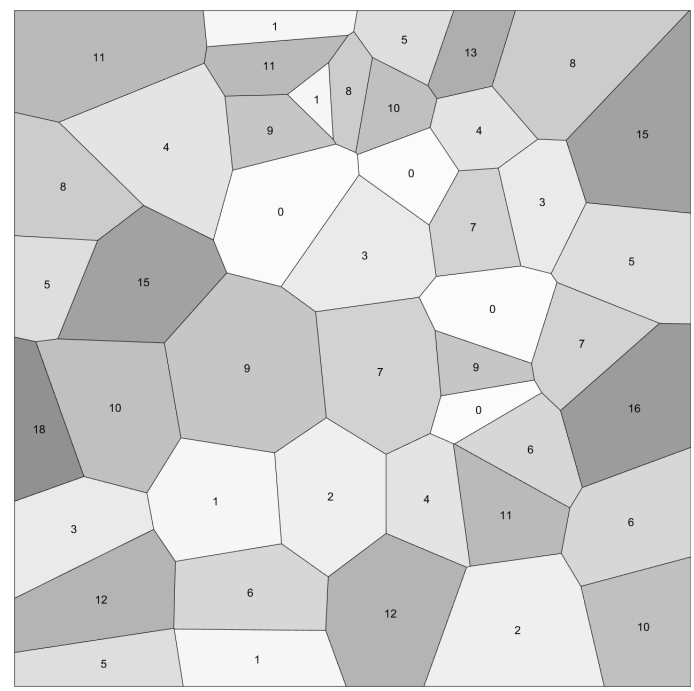

Figure 8: Ages of cells after 19 years

For experiment (rh2) habitat constraints were relaxed and solutions compared with the nominal results. Figure 9 shows that fire hazard is not further reduced significantly. In other words the local habitat constraint has not diminished significantly the fuel reduction objective. Not shown is that collectively for the 23 scenarios, the local habitat connectivity constraint is not satisfied in nearly $50 \%$ of cases.

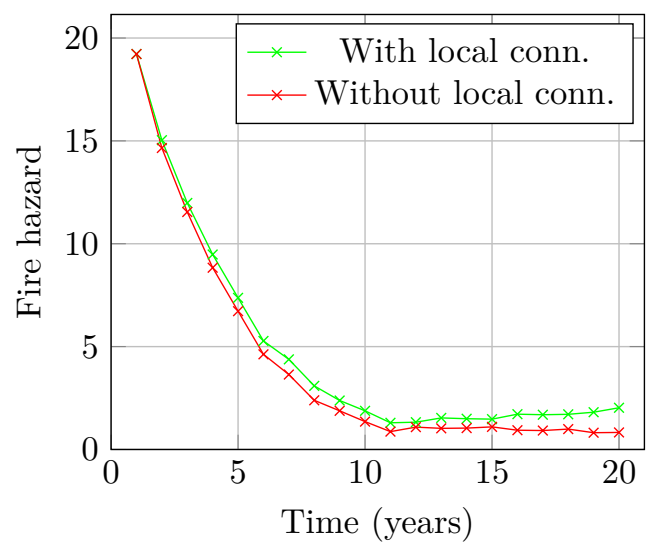

Figure 9: Mean fire hazard when local connectivity is not a requirement. Hazard does not change significantly but local connectivity is violated many times (210 times on 437 runs of the model).

In experiment (rh3), out of the 23 scenarios three were infeasible when solved with the myopic approach. As habitat constraints are not included, the source of infeasibility is a result of constraint (6) requiring vegetation exceeding the fuel age of the parameter maxTFI to be burnt. In some scenarios situations arise in which the amount to be burnt in one year is greater than that allowed by the budget (constraint (2)). 


\begin{tabular}{c|c|c} 
Year & Long term & Myopic \\
\hline 16 & 0.936 & 2.258 \\
17 & 0.920 & 2.076 \\
18 & 0.991 & 1.899 \\
19 & 0.815 & 2.036 \\
20 & 0.828 & 2.045
\end{tabular}

Table 1: Mean fire hazard in the last years of simulation, long term rolling horizon window versus myopic approach.

Table 1 reports the results obtained for the last years of the planning horizon, when the effect of initial values have diminished. Even removing the scenarios in which the myopic approach was infeasible, the short-term plans yielded results much worse than that obtained with a longer planning horizon.

In experiment (L1) all three solutions have fire hazard going to zero. Habitat values are shown in Table 2.

\begin{tabular}{c|c|c|c} 
Year & Solution 1 & Solution 2 & Solution 3 \\
\hline 10 & 6.502 & 7.804 & 5.675 \\
11 & 5.539 & 7.538 & 5.122 \\
12 & 4.987 & 6.449 & 4.401 \\
13 & 4.912 & 6.456 & 4.311 \\
14 & 4.699 & 6.312 & $\mathbf{4 . 1 2 2}$ \\
15 & 4.64 & 6.399 & 5.033 \\
16 & 4.604 & 6.3 & 4.953 \\
17 & 4.822 & 6.327 & 4.865 \\
18 & 5.109 & 6.287 & 4.785 \\
19 & 5.174 & 6.316 & 4.844 \\
20 & 5.091 & $\mathbf{6 . 2 8 4}$ & 5.025
\end{tabular}

Table 2: Yearly habitat values for three different solutions, all with the same fire hazard. In boldface the minimum habitat value for solutions 2 and 3 .

Without compromising the reduction in fuel hazard, a comparison of Solution 2 and Solution 1 reveals that the model can yield significant improvements (more than a third) in habitat quality. In fact, it is possible that a fuel reduction plan such as solution 3 could yield habitat quality more than $50 \%$ below its optimal value. There is thus a large interval of habitat values that can result with the same fuel hazard value. This opportunity for optimising habitat should not be neglected.

The state of the landscape for experiment (L2) is shown in Figure 10 for the last year of the planning period. It can be seen that the model makes use of the new definition of neighbours, as fuel load is accumulated in burn units that are geographically adjacent in the north-south direction but were only defined as neighbours in the eat-west direction, and thus do not pose a high fire hazard. 


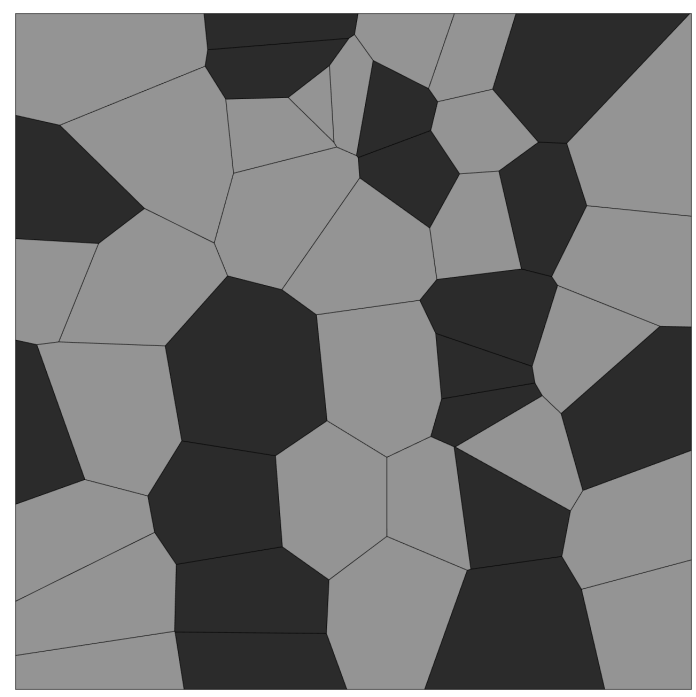

Figure 10: Solution in the last year of simulation with dark units reflecting burn units that have old fuel (their age is older than 10).

\section{Conclusion}

We presented a mixed integer programming model for a landscape divided into polygons representing realistic treatement units. The model aims to reduce the adjacency of high fuel load areas. We show that adopting a medium-term approach to fuel reduction using our model is much more effective than adopting a myopic approach. In this latter case it frequently arises that fuel reduction targets cannot be met within budget constraints.

There are ecological consequences from prescribed burning. We considered habitat quality for invertebrates on a heathland landscape and also a hypothetical species that had a preference for hazardous vegetation. We showed that a significant range of habitat quality outcomes can be obtained without compromising the optimal fuel load goal. It is sensible therefore for habitat considerations to be included in fuel reduction plans. We showed that this can be achieved for invertebrates by requiring the habitat quality in the neighbourhood of a planned burn be at least as good as the habitat quality of the area to be burnt. Such a constraint is applicable for any species with a small home range. For species with large home ranges it might be enough only take into account landscape-level habitat quality we included. This consideration of local and global habitat differs from previous work. We also imposed some ecological requirements in the form of minimum and maximum tolerable fire intervals for the vegetation used in previous work. For any particular landscape, factors such as topology and prevailing winds will determine connectnedness between high fuel load areas. We have illustrated that this can be handled with a redefinition of the neighbourhood of each treatment unit. In fact, where fire spread is predominantly in certain directions geographically, adjacent treatment units might not be in the same neighbourhood from a fuel connectedness perspective. This creates opportunities for maintaining habitat quality for species requiring older vegetation without compromising fuel reduction plans. Computational effort in solving our mixed integer programming model can be considerable and limiting. Both the rolling horizon and the lexicographic approach offer possibilities of achieving solutions in times suitable for using in a workshop situation. Whichever method is the most appropriate for a given problem the model should be solved again each year to take into account 
unplanned fires or unexpected rates of vegetation senescence that might have occurred over the previous year.

\section{Acknowledgement}

This work has been supported by the Marie Skłodowska-Curie RISE H2020 project GEO-SAFE (691161), Government of Spain (MTM2015-65803-R), the Goverment of Madrid (S2013/ICE2845 CASI-CAM-CM) and the UCM grant (CT27/16-CT28/16). The authors are very grateful to an anonymous reviewer whose comments and suggestions have helped considerably in enhancing this paper.

\section{References}

[1] James K. Agee and Carl N. Skinner, Basic principles of forest fuel reduction treatments, Forest ecology and management 211 (2005), no. 1, 83-96.

[2] Alan A. Ager, Nicole M. Vaillant, and Andrew McMahan, Restoration of fire in managed forests: A model to prioritize landscapes and analyze tradeoffs, Ecosphere 4 (2013), no. 2, $1-19$.

[3] Fermín J. Alcasena, Alan A. Ager, Michele Salis, Michelle A. Day, and Cristina Vega-García, Optimizing prescribed fire allocation for managing fire risk in central catalonia, Science of The Total Environment 621 (2018), 872 - 885.

[4] Jeff Bezanson, Alan Edelman, Stefan Karpinski, and Viral B. Shah, Julia: A fresh approach to numerical computing, SIAM Review 59 (2017), no. 1, 65-98.

[5] Matthias M. Boer, Rohan J. Sadler, Roy S. Wittkuhn, Lachlan McCaw, and Pauline F. Grierson, Long-term impacts of prescribed burning on regional extent and incidence of wildfiresevidence from 50 years of active fire management in $S W$ Australian forests, Forest Ecology and Management 259 (2009), no. 1, 132-142.

[6] Sarah Brown, Michael Clarke, and Rohan Clarke, Fire is a key element in the landscape-scale habitat requirements and global population status of a threatened bird: The Mallee Emu-wren (Stipiturus mallee), Biological Conservation 142 (2009), no. 2, 432-445.

[7] Neil Burrows, Linking fire ecology and fire management in south-west Australian forest landscapes, Forest Ecology and Management 255 (2008), no. 7, 2394-2406.

[8] Henry Carey and Martha Schumann, Modifying wildfire behavior-the effectiveness of fuel treatments, The Forest Trust (2003), 16.

[9] David Cheal, Growth stages and tolerable fire intervals for Victoria's native vegetation data sets. Fire and Adaptive Management Report 84, 2010.

[10] Woodam Chung, Optimizing fuel treatments to reduce wildland fire risk, Current Forestry Reports 1 (2015), no. 1, 44-51.

[11] Julian Di Stefano, Michael A. McCarthy, Alan York, Thomas J. Duff, Jacqui Slingo, and Fiona Christie, Defining vegetation age class distributions for multispecies conservation in fire-prone landscapes, Biological Conservation 166 (2013), 111 - 117. 
[12] Iain Dunning, Joey Huchette, and Miles Lubin, Jump: A modeling language for mathematical optimization, SIAM Review 59 (2017), no. 2, 295-320.

[13] Paulo M. Fernandes and Hermínio S. Botelho, A review of prescribed burning effectiveness in fire hazard reduction, International Journal of wildland fire 12 (2003), no. 2, 117-128.

[14] Mark A. Finney, Design of regular landscape fuel treatment patterns for modifying fire growth and behavior, Forest Science 47 (2001), no. 2, 219-228.

[15] Mark A. Finney, A computational method for optimising fuel treatment locations, International Journal of Wildland Fire 16 (2008), no. 6, 702-711.

[16] Mark A. Finney, Rob C. Seli, Charles W. McHugh, Alan A. Ager, Bernhard Bahro, and James K. Agee, Simulation of long-term landscape-level fuel treatment effects on large wildfires, International Journal of Wildland Fire 16 (2008), no. 6, 712-727.

[17] U.S.G.A.O. GAO, Wildland fire management: Additional actions required to better identify and prioritize lands needing fuels reduction: Report to congressional requesters., Washington, D.C. (441 G St., NW, Washington 20548): The Office. (2003).

[18] LLC Gurobi Optimization, Gurobi optimizer reference manual, 2018.

[19] John Hof and Philip Omi, Scheduling removals for fuels management, USDA Forest Service Proceedings RMRS-P-29, Citeseer, 2003, pp. 367-378.

[20] David A Keith, W Lachie McCaw, and Robert J Whelan, Fire regimes in Australian heathlands and their effects on plants and animals, Flammable Australia: the fire regimes and biodiversity of a continent. Cambridge University Press, Cambridge (2002), 199-237.

[21] Young-Hwan Kim, Pete Bettinger, and Mark A. Finney, Spatial optimization of the pattern of fuel management activities and subsequent effects on simulated wildfires, European Journal of Operational Research 197 (2009), no. 1, 253-265.

[22] Karen J. King, Ross A. Bradstock, Geoffrey J. Cary, Joanne Chapman, and Jon B. MarsdenSmedley, The relative importance of fine-scale fuel mosaics on reducing fire risk in south-west Tasmania, Australia, International Journal of Wildland Fire 17 (2008), no. 3, 421-430.

[23] Karen J. King, Geoffrey J. Cary, Ross A. Bradstock, Joanne Chapman, Adrian Pyrke, and Jonathon B. Marsden-Smedley, Simulation of prescribed burning strategies in south-west Tasmania, Australia: effects on unplanned fires, fire regimes, and ecological management values, International Journal of Wildland Fire 15 (2006), no. 4, 527-540.

[24] Craig Loehle, Applying landscape principles to fire hazard reduction, Forest Ecology and management 198 (2004), no. 1, 261-267.

[25] Josephine MacHunter, Peter Menkhorst, and Richard H. Loyn, Towards a process for integrating vertebrate fauna into fire management planning, Arthur Rylah Institute for Environmental Research, Department of Sustainability and Environment, 2009.

[26] James P. Minas, John W. Hearne, and David L. Martell, A spatial optimisation model for multi-period landscape level fuel management to mitigate wildfire impacts, European Journal of Operational Research 232 (2014), no. 2, 412-422. 
[27] Dung Tuan Nguyen, Develop a multistage stochastic program with recourse for scheduling prescribed burning based fuel treatments with consideration of future wildland fires and fire suppressions, Ph.D. thesis, Colorado State University. Libraries, 2015.

[28] Tiago M. Oliveira, Ana M.G. Barros, Alan A. Ager, and Paulo M. Fernandes, Assessing the effect of a fuel break network to reduce burnt area and wildfire risk transmission, International Journal of Wildland Fire 25 (2016), no. 6, 619-632.

[29] Trent D. Penman, Fiona J. Christie, AN Andersen, Ross A. Bradstock, GJ Cary, Meredith K. Henderson, Owen Price, Cuong Tran, Glenda M. Wardle, RJ Williams, et al., Prescribed burning: how can it work to conserve the things we value?, International Journal of Wildland Fire 20 (2011), no. 6, 721-733.

[30] Ramya Rachmawati, Melih Ozlen, John Hearne, and Karin Reinke, Fuel treatment planning: Fragmenting high fuel load areas while maintaining availability and connectivity of faunal habitat, Applied Mathematical Modelling 54 (2018), 298 - 310.

[31] Ramya Rachmawati, Melih Ozlen, Karin J Reinke, and John W Hearne, A model for solving the prescribed burn planning problem, SpringerPlus 4 (2015), no. 1, 1-21.

[32] Ramya Rachmawati, Melih Ozlen, Karin J. Reinke, and John W. Hearne, An optimisation approach for fuel treatment planning to break the connectivity of high-risk regions, Forest Ecology and Management 368 (2016), 94 - 104.

[33] Bronwyn Rayfield, David Pelletier, Maria Dumitru, Jeffrey A. Cardille, and Andrew Gonzalez, Multipurpose habitat networks for short-range and long-range connectivity: a new method combining graph and circuit connectivity, Methods in Ecology and Evolution (2015).

[34] Mikael Rönnqvist, Sophie D'Amours, Andres Weintraub, Alejandro Jofre, Eldon Gunn, Robert G. Haight, David Martell, Alan T Murray, and Carlos Romero, Operations research challenges in forestry: 33 open problems, Annals of Operations Research 232 (2015), no. 1, 11-40.

[35] Adam Rytwinski and Kevin A. Crowe, A simulation-optimization model for selecting the location of fuel-breaks to minimize expected losses from forest fires, Forest ecology and management 260 (2010), no. 1, 1-11.

[36] Lucy A. Salazar and Armando González-Cabán, Spatial relationship of a wildfire, fuelbreaks, and recently burned areas, Western Journal of Applied Forestry 2 (1987), no. 2, 55-58.

[37] Darren M. Southwell, Alex M. Lechner, Terry Coates, and Brendan A. Wintle, The sensitivity of population viability analysis to uncertainty about habitat requirements: Implications for the management of the endangered Southern Brown Bandicoot, Conservation Biology 22 (2008), no. 4, 1045-1054.

[38] Barbara A. Strom and Peter Z Fulé, Pre-wildfire fuel treatments affect long-term ponderosa pine forest dynamics, International Journal of Wildland Fire 16 (2007), no. 1, 128-138.

[39] Matthew P. Thompson, Phil Bowden, April Brough, Joe H. Scott, Julie Gilbertson-Day, Alan Taylor, Jennifer Anderson, and Jessica R. Haas, Application of wildfire risk assessment results to wildfire response planning in the southern sierra nevada, california, usa, Forests 7 (2016), no. 3 . 
[40] Tyron J. Venn and David E. Calkin, Accommodating non-market values in evaluation of wildfire management in the united states: challenges and opportunities, International Journal of Wildland Fire 20 (2011), no. 3, 327-339.

[41] Yu Wei, Optimize landscape fuel treatment locations to create control opportunities for future fires, Canadian Journal of Forest Research 42 (2012), no. 6, 1002-1014.

[42] Yu Wei and Yehan Long, Schedule fuel treatments to fragment high fire hazard fuel patches, Mathematical and Computational Forestry \& Natural-Resource Sciences (MCFNS) 6 (2014), no. $1,1-10$.

[43] Yu Wei, Douglas Rideout, and Andy Kirsch, An optimization model for locating fuel treatments across a landscape to reduce expected fire losses, Canadian Journal of Forest Research 38 (2008), no. 4, 868-877. 